\title{
ORIENTED TORAL KNOTS LATTICES
}

\author{
RUSSELL B. WALKER
}

\begin{abstract}
The common intersections between two sets of toral knots, having differing toral knot types and differing sequences of orientations, are removed. The remaining arc-segments are reconnected in a prescribed manner. In closed form, the number of "resulting loops" is provided.
\end{abstract}

A question concerning two foliations of the 2-torus, one containing $(p, q)$-Reeb components, the other $(r, s)$-Reeb components, leads in a natural way to a problem involving toral knot splicing and recombination $[2,3]$ :

One set of $n$ pairwise nonintersecting type $(p, q)$ toral knots (the knot wraps $p$ times longitudinally while $q$ times meridionally) is given the orientation $o^{1} \in$ $\prod_{i=1}^{n}\{+1,-1\}$. Here +1 refers to one possible $(p, q)$-“direction", -1 the other. Likewise, a set of $m$ type $(r, s)$-toral knots $((r, s) \neq(p, q))$ is given the orientation, $o^{2} \in \prod_{i=1}^{m}\{+1,-1\}$. The two sets are assumed to have the minimum number of intersection points, $n m|q r-p s|$. These points are removed and the remaining intervals reconnected according to Figure 1. A resulting system of toral knots and null-homotopic loops are formed by this procedure.

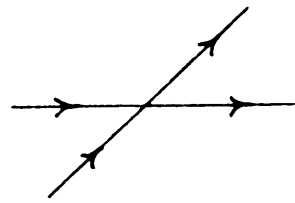

becomes

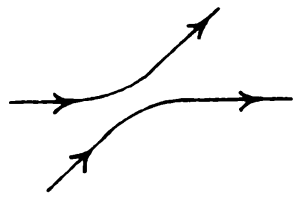

FIGURE 1

Problem. What is the number and type of these resulting toral knots and how many such loops exist?

One imagines a "toral city" having two sets of one-way toral knot "streets". If resident "drivers" are required to turn at every corner, how many possible "routes" exist and what are their types. Of course, the sequence as well as the number of "left onlys" and "right onlys" will effect the question's answer. In this note, an answer in closed form is provided

Oriented toral knot systems. The described collection of oriented $(p, q)$ - and $(r, s)$-knots is an oriented toral knot lattice and is denoted by $L$. The associated

Received by the editors October 5, 1981.

1980 Mathematics Subject Classification. Primary 05C10; Secondary 57M15.

Key words and phrases. Toral knot, oriented lattices.

(c) 1983 American Mathematical Society $0002-9939 / 82 / 0000-0696 / \$ 02.00$ 
collection of knots and loops which result from the prescribed splicing and recombination, denoted by $\tilde{L}$, is a resulting toral knot system. Let $K(\tilde{L})$ be the number of resulting toral knots. Each such knot has the same toral knot type, $(u(\tilde{L}), v(\tilde{L}))$. $N(\tilde{L})$ is the number of null-homotopic loops within $\tilde{L} . N_{0}(\tilde{L})$ of these loops are minimal; such loops contain four reconnection points.

$K(\tilde{L}),(u(\tilde{L}), v(\tilde{L}))$, and $N(\tilde{L})$ are sought in terms of $o^{1}=o^{\prime}(L)$ and $o^{2}=o^{2}(L)$. The key observation is that $\tilde{L}$ may be reduced by first counting and then removing the minimal loops. Large null-homotopic loops become minimal, these are counted and then removed until no null-homotopic loops remain. This sequence of reductions may be made without effecting $K(\tilde{L})$ or $(u(\tilde{L}), v(\tilde{L}))$ and leaves a simpler computation.

The reduction procedure is as follows: each consecutive pair of like-oriented toral knots from the lattice $L$ is removed and replaced by a single knot having the same type and orientation lying midway between the original two. After the same prescribed splicing and reconnection, this first-reduction, $L_{1}$, becomes $\tilde{L}_{1}$. Further reductions and associated systems are designated $L_{2}, \tilde{L}_{2}, L_{3}, \tilde{L}_{3}, \ldots$ The orientations at each stage are $o^{1}\left(L_{2}\right), o^{2}\left(L_{2}\right), o^{1}\left(L_{3}\right), o^{2}\left(L_{3}\right), \ldots$

EXAMPLE 1. $(p, q)=(1,0),(r, s)=(0,1), o^{1}(L)=(1,-1), o^{2}(L)=(1,-1), K(\tilde{L})$ $=0, N(\tilde{L})=N_{0}(\tilde{L})=2$. The first reduction is empty.

EXAMPLE 2. $(p, q)=(1,0),(r, s)=(0,1), o^{1}(L)=(1,1,-1,1,-1,-1,1,-1)$, $o^{2}(L)=(1,1,-1,1,1,-1,1,-1,-1,-1,1,-1,1), K(\tilde{L})=3$ with $(u(\tilde{L}), v(\tilde{L}))=$ $(0,1), N(\tilde{L})=26$, and $N_{0}(\tilde{L})=24$. After the first reduction, $K\left(\tilde{L}_{1}\right)=3$ with $\left(u\left(\tilde{L}_{1}\right), v\left(\tilde{L}_{1}\right)\right)=(0,1), N\left(\tilde{L}_{1}\right)=2$, and $N_{0}\left(\tilde{L}_{1}\right)=2$. The first reduction of $L_{1}$ has no resulting null-homotopic loops: $K\left(\tilde{L}_{2}\right)=3$ with $\left(u\left(\tilde{L}_{2}\right), v\left(\tilde{L}_{2}\right)\right)=(0,1), N\left(\tilde{L}_{2}\right)$ $=N_{0}\left(\tilde{L}_{2}\right)=0$.

The following lemma provides the relationship between knot and loop counts before and after reduction.

LEMMA. If $N_{0}(\tilde{L}) \neq 0$, then $K\left(\tilde{L}_{1}\right)=K(\tilde{L}),\left(u\left(\tilde{L}_{1}\right), v\left(\tilde{L}_{1}\right)\right)=(u(\tilde{L}), v(\tilde{L}))$, and $N\left(\tilde{L}_{1}\right)=N(\tilde{L})-N_{0}(\tilde{L})$.

Proof. Consecutive pairs of like-oriented $(p, q)$-toral knots of $L$, in a sense, "trap" a resulting knot or large null-homotopic loop of $\tilde{L}$ until the knot or loop encounters a like-oriented pair of $(r, s)$-toral knots. This new pair traps the knot or loop, containing its $(p, q)$-“oscillations", until like-oriented $(p, q)$-knots are encountered.

However, it one of the two sets of knots of $L$ are like-oriented, resulting loops and knots cannot make such transitions from one set to the other. But then, $N_{0}(\tilde{L})=0$, for minimal loops are formed only between two pairs of opposite-oriented knots (see Figure 6).

The reduced system, $\tilde{L}_{1}$, contains toral knots and large null-homotopic loops which shadow these oscillations and transitions. Thus each toral knot of type $(u(\tilde{L}), v(\tilde{L}))$ in $\tilde{L}$ has a representative in $\tilde{L}_{1}$, and vise versa. $K\left(\tilde{L}_{1}\right)=K(\tilde{L})$ and $\left(u\left(\tilde{L}_{1}\right), v\left(\tilde{L}_{1}\right)\right)=(u(\tilde{L}), v(\tilde{L}))$.

Likewise, the larger loops of $L$ have representatives in $\tilde{L}_{1}$. The minimal loops are not shadowed and are lost in the reduction procedure; $N\left(\tilde{L}_{1}\right)=N(\tilde{L})-N_{0}(\tilde{L})$ as desired. 


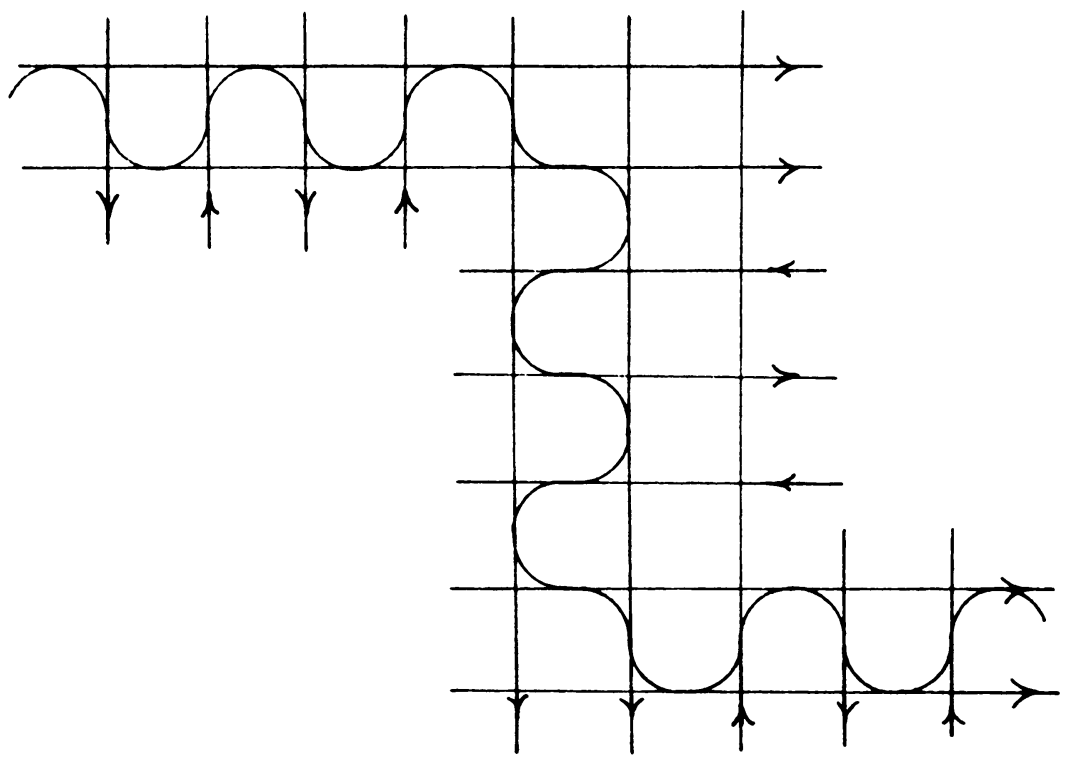

FIGURE 2

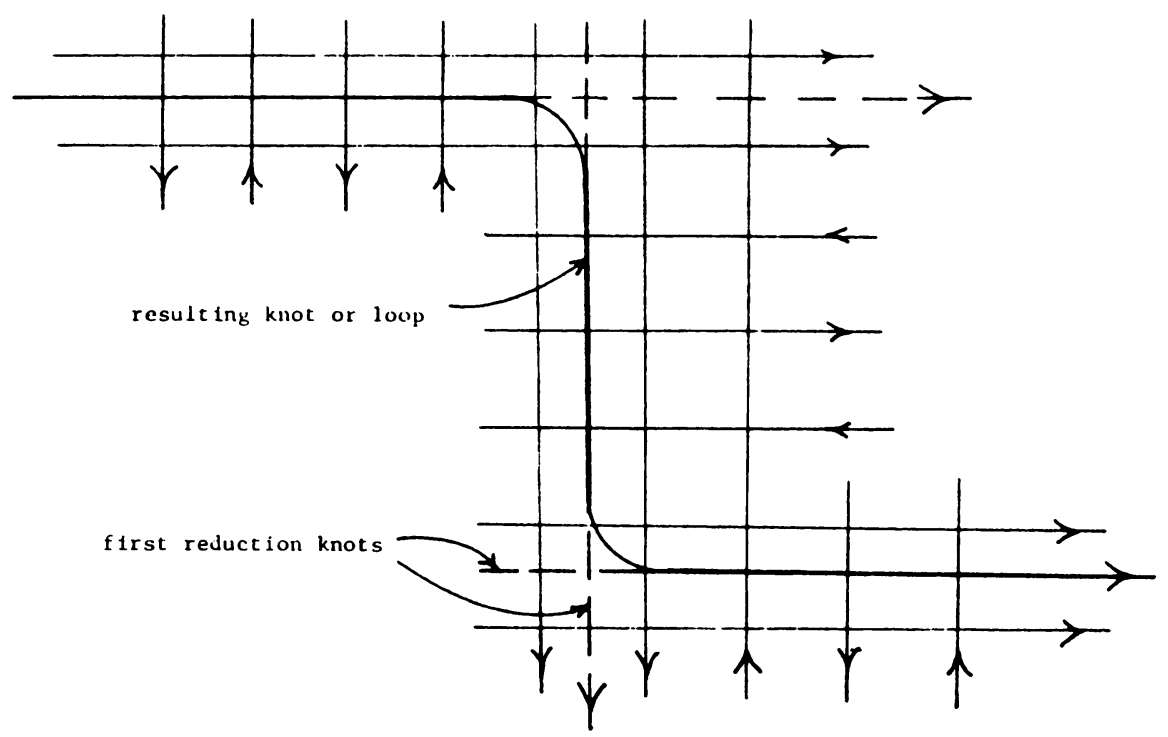

Figure 3

The $k$ th-reduction, $L_{k}$, has $n_{k}$ type $(p, q)$-knots and $m_{k}$ type $(r, s)$-knots. $L_{k}$ is a final reduction if $N\left(\tilde{L}_{k}\right)=0$ while $N\left(\tilde{L}_{k-1}\right) \neq 0$. Note that if $N\left(\tilde{L}_{k}\right) \neq 0$ for some $k$, $N_{0}\left(\tilde{L}_{k}\right) \neq 0$. The final reduction is denoted $L_{f}$ with associated system $\tilde{L}_{f} . L_{f}$ has $n_{f}$ $(p, q)$-knots and $m_{f}(r, s)$-knots. 
To facilitate the counting of minimal loops, the number of consecutive oppositely oriented pairs of the $k$ th reduction must be counted. The coordinates of the two orientations of $L_{k}$ are denoted

$$
o^{1}\left(L_{k}\right)=\left(o_{1}^{1}\left(L_{k}\right), \ldots, o_{n_{k}}^{1}\left(L_{k}\right)\right) \text { and } o^{2}\left(L_{k}\right)=\left(o_{1}^{2}\left(L_{k}\right), \ldots, o_{m_{k}}^{2}\left(L_{k}\right)\right) \text {. }
$$

where $o_{n_{k}+1}^{1}\left(L_{k}\right)$ is identified with $o_{1}^{1}\left(L_{k}\right)$ and $o_{m_{k}+1}^{2}\left(L_{k}\right)$ is identified with $o_{1}^{2}\left(L_{k}\right)$. Then for $i=1,2$ let

$$
o_{+}^{\prime}(k)=\sharp\left\{o_{j}^{i}\left(L_{k}\right): o_{j}^{\prime}\left(L_{k}\right)=-1 \text { and } o_{j+1}^{\prime}\left(L_{k}\right)=+1\right\},
$$

and

$$
o_{-}^{i}(k)=\#\left\{o_{j}^{i}\left(L_{k}\right): o_{j}^{i}\left(L_{k}\right)=+1 \text { and } o_{j+1}^{i}\left(L_{k}\right)=-1\right\}
$$

THEOREM. Let $u=p \sum_{j=1}^{n} o_{j}^{\prime}(L)+r \sum_{j=1}^{m} o_{j}^{2}(L)$ and $v=q \sum_{j=1}^{n} o_{j}^{l}(L)+$ $s \sum_{j=1}^{m} o_{j}^{2}(L)$. Then if $\min \left\{n_{f}, m_{f}\right\} \neq 0, K(\tilde{L})=$ G.C.D. $(u, v)$ and $(u(\tilde{L}), v(\tilde{L}))=$ $(u / K(\tilde{L}), v / K(\tilde{L}))$; otherwise, $K(L)=\max \left\{n_{f}, m_{f}\right\}$. Furthermore, in both cases

$$
N(\tilde{L})=|q r-p s| \sum_{h=0}^{k_{0}}\left[o_{+}^{1}(k) o_{-}^{2}(k)+o_{-}^{1}(k) o_{+}^{2}(k)\right]
$$

where $L_{k_{0}}=L_{f}$.

Proof (Toral knots of $\tilde{L}$ ). By the lemma, $K(\tilde{L})=K\left(\tilde{L}_{f}\right)$. If $\min \left\{n_{f}, m_{f}\right\}=0$. then $K\left(\tilde{L}_{f}\right)$ is exactly the number of $(p, q)$ - or $(r, s)$-knots which remain.

Assume $\min \left\{n_{f}, m_{f}\right\}=0$. Either the $(p, q)$ - or $(r, s)$-knots of $L_{f}$ are likeoriented. For otherwise, $\tilde{L}_{f}$ contains a minimal loop (see Figure 6 ). So assume the $(r, s)$-knots are like-oriented. Then the toral knots of $\tilde{L}_{f}$, in a sense, run in parallel, consecutively crossing $(p, q)$-knots. Each contains the same number of reconnection points, two at each crossing. Thus, $K\left(\tilde{L}_{f}\right)$ is the total number of reconnection points, or $2 n_{f} m_{f}|q r-p s|$, divided by the number of reconnection points lying on each knot of $\tilde{L}_{f}$. This second number is $2 n_{f}|v(\tilde{L}) p-u(\tilde{L}) q|$, or twice the number of times a type $(u(\tilde{L}), v(\tilde{L}))$-knot crosses a $(p, q)$-knot.

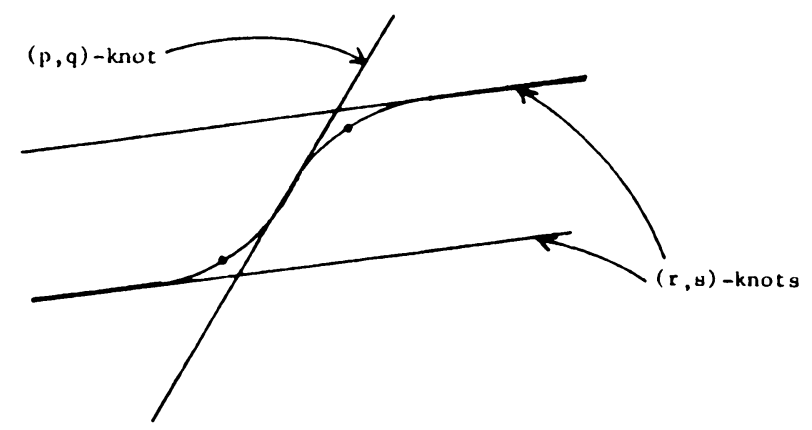

Figure 4 
For the purposes of computing $(u(\tilde{L}), v(\tilde{L}))$, the set of $(p, q)$-knots of $L_{f}$ are further reduced in the usual manner until they too are like-oriented. The new system is denoted $\tilde{L}_{0}$, and has $n_{0}(p, q)$-knots and $m_{0}(r, s)$-knots. So $m_{0}=m_{f}$. If $n_{0}=m_{0}=1$, resulting knots in $\tilde{L}_{0}$ have type $(p+r, q+s) /$ G.D.C. $(p+r, q+s)$. This, because they seem to climb parallelogram-like stairs having slope (in the covering space, $\left.\mathbf{R}^{2}\right),(p+r) /(q+s)$.

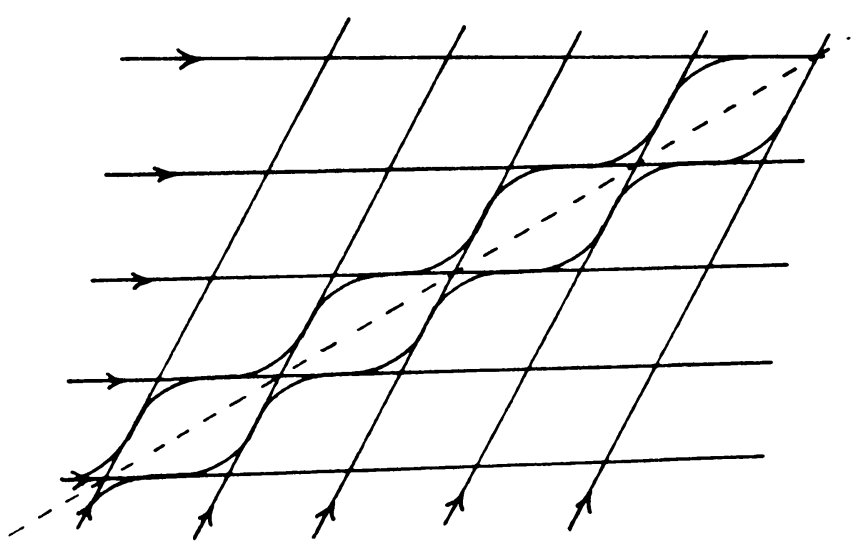

Figure 5

Each additional $(p, q)$ - or $(r, s)$-knot in $\tilde{L}_{0}$ has the effect of an extra push in the $(p, q)$ - or $(r, s)$-direction (up in $\mathbf{R}^{2}$ ). Thus

$$
(u(\tilde{L}), v(\tilde{L}))=\frac{\left(n_{0} p+m_{0} r, n_{0} q+m_{0} s\right)}{\text { G.C.D. }\left(n_{0} p+m_{0} r, n_{0} q+m_{0} s\right)} .
$$

The numerator is precisely $(u, v)$; call the denominator, $K$. Thus

$$
\begin{aligned}
K(\tilde{L}) & =\frac{2 n_{f} m_{f}|q r-p s|}{2 n_{f}|v p-u q| / k}=\frac{K m_{f}|q r-p s|}{\left|\left(q n_{0}+s m_{0}\right) p-\left(p n_{0}+r m_{0}\right) q\right|} \\
& =\frac{K m_{f}|q r-p s|}{m_{0}|p s-q r|}=K
\end{aligned}
$$

since $m_{f}=m_{0}$.

(Null homotopic loops). The minimal loops result in two manners:
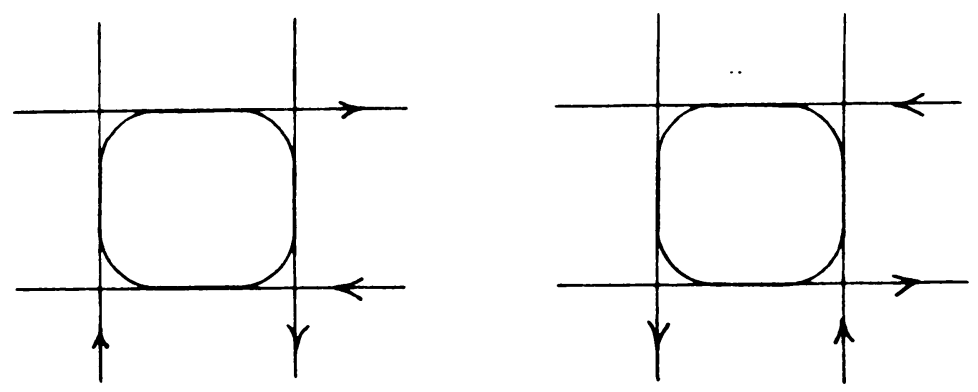

FIGURE 6 
So each time a pair of consecutive $(p, q)$-knots with orientations $(+1,1)$ intersects a pair of $(r, s)$-knots with orientations $(-1,+1)$, a minimal loop is formed. Likewise for the $(-1,+1),(+1,-1)$ overlaps. Thus the number of minimal loops at the $k$ th-stage is $|q r-p s|\left(o_{+}^{1}(k) o_{-}^{2}(k)+o_{-}^{1}(k) o_{+}^{2}(k)\right)$ as desired.

(REMARK. The subject matter of this paper is related to graph theory and has been called Topological Combinatorics. For related work see Steven Fisk [1].)

\section{REFERENCES}

1. Steven Fisk, Geometric coloring theory, Adv. in Math. 24 (1977), 298-340.

2. Russell Walker. Morse and generic contact between foliations, Trans. Amer. Math. Soc. 254 (1979), 265-281.

3. Contact between foliations. Thesis, University of California, Berkeley, 1977.

Department of Mathematics, Montana State University, Bozeman, Montana 59717 